\title{
A Constructive Method for Exploiting Code Motion
}

\author{
Luiz C. V. dos Santos' ${ }^{1}$, M.J.M. Heijligers, C.A.J. van Eijk, J.T.J. van Eijndhoven and J.A.G. Jess \\ Design Automation Section, Eindhoven University of Technology \\ P.O.Box 513, 5600 MB Eindhoven, The Netherlands
}

\begin{abstract}
In this paper we address a resource-constrained optimization problem for behavioral descriptions containing conditionals. In high-level synthesis of ASICs or in code generation for ASIPs, most methods use greedy choices in such a way that the search space is limited by the applied heuristics. For example, they might miss opportunities to optimize across basic block boundaries when treating conditional execution. We propose an approach based on local search and present a constructive method to allow unrestricted types of code motion, while keeping optimal solutions in the search space. A code-motion pruning technique is presented for cost functions optimizing schedule lengths. A technique for treating concurrent flows of execution is also described.
\end{abstract}

\section{Introduction}

In the high-level synthesis of an application-specific integrated circuit (ASIC) or in the code generation for an application-specific instruction set processor (ASIP), four main difficulties have to be faced when conditionals are present in the behavioral description:

a) the NP-completeness of the resource-constrained scheduling problem itself.

b) the limited parallelism of operations enclosed by basic blocks, as they might not use all available resources completely.

c) the possibility of state explosion because the number of paths may explode in the presence of conditionals.

d) the possibility of state expansion due to limited resource sharing of mutually exclusive operations (which is limited by the timely availability of test results).

Most methods apply different heuristics for each subproblem (BB scheduling, code motion, code size reduction) as if they were independent. An heuristic is used to decide the order of the operations during scheduling (like the many flavors of priority lists), another to decide whether a particular code motion is worth doing $[6,17]$, yet another for a reduction on the number of states [18]. As a result, these approaches might miss optimal solutions.

We propose a formulation to encode potential solutions for the interdependent subproblems. We show that optimal solutions are kept in the search space. The formulation abstracts from the linear-time model and allows us to concentrate on the order of operations and availability of resources. Figure 1 shows the outline of our approach. We

\footnotetext{
1. On leave from INE, Fed. Univ. of Santa Catarina, Brazil. Partially supported by CNPq (Brazil) under fellowship award n. 200283/94-4
}

have a solution explorer which is based on a local search algorithm [15]. The constructor is driven by a permutation $\Pi$ of the operations in the data flow graph. The explorer handles encoded solutions and uses a solution constructor to evaluate their cost. A code-motion pruning is embedded in the constructor to reduce the search space.

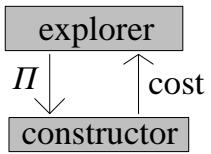

Figure 1 - An outline of the approach

The main contributions of our approach are:

a) A method to encode BB scheduling and code motion as an unified problem such that unrestricted code motions can be induced;

b) A code-motion pruning technique which preserves optimal solutions in the search space;

c) A technique for treating concurrent flows of execution when a single flow of control is targeted.

\section{The Problem}

Definition 1: A control data flow graph $\mathrm{DFG}=(\mathrm{U}, \mathrm{E})$ is a directed graph where the nodes represent operations and the edges represent the dependencies between them. We assume that the DFG contains conditional constructs.

Definition 2: An execution condition is associated with every operation, represented as a boolean function, here called a predicate. An execution instance (EXI) is a set of operations executed under a given predicate.

Definition 3: A basic block (BB) is a set of operations which all have the same execution condition.

Definition 4: A basic block control flow graph $\mathrm{BBCG}=$ $(\mathrm{V}, \mathrm{F})$ is a directed graph where the nodes represent basic blocks and the edges represent the flow of control. We allow that an operation initially associated with a given $\mathrm{BB}$ be "moved" to another BB; this is called code motion.

Definition 5: Each traversal in the BBCG from an input node to an output node such that only BBs which execute under a same predicate are visited defines a path. A path defines a sequence of BBs which enclose all the operations belonging to an EXI of the DFG. Each path in the BBCG corresponds to exactly one EXI in the DFG.

Optimization problem (OP): Given a number $K$ of functional units and an acyclic DFG, find a control sequence represented by a state machine graph, in which precedence constraints of the DFG are obeyed and the resource constraints are satisfied for each functional unit type, such that a cost function $C$ is minimized. 
Our motivation to address this resource-constrained problem is due to the increasing interest to find the balance between architectural synthesis and code generation techniques (e.g. application-domain ASIPs).

General instances of the $O P$ : The method presented in this paper addresses instances of the OP for arbitrary cost functions which can be extended to include not only schedule length, but also issues like register and interconnect usage, and number of states. This is convenient especially in late phases of a design flow, where optimization has to take several design issues into account.

Particularinstances of the $O P$ : In early phases of a design flow, the optimization objectives are dictated by the realtime requirements of embedded systems design. The slowest possible execution time of a piece of code must meet realtime constraints [4]. At the other hand, as these early phases tend to be iterated several times, runtime efficiency is imperative. For these reasons we propose a pruning technique to tackle instances of the OP for which the cost function can be written as $C=f\left(T_{1}, T_{2}, \ldots, T_{n}\right)$, where $T_{i}$ is the schedule length of the $\mathrm{i}^{\text {th }}$ path in the BBCG and $f$ is a monotonically increasing function. This pruning guarantees the presence of optimal solutions in the search space in terms of schedule lengths (see [8] and proof in Appendix A).

\section{Related Work}

In path-based scheduling (PBS) $[3,2]$ a so-called asfast-as-possible (AFAP) schedule is found for each path independently, provided that a fixed order of operations be chosen in advance. Due to the fixed order and to the fact that scheduling is cast as a clique covering problem on an interval graph, code motions resulting in speculative execution are not allowed. Thus, the method has limited capability of exploiting parallelism with complex control flow [13]. This limitation is released in tree-based scheduling (TBS) [10], by allowing boosting and duplication code motions.

Condition vector list scheduling (CVLS) [18] allows code motion and supports speculative execution. However, the underlying mutual exclusion representation is limited [1].

In Trace-scheduling (TS) [6] a main path (trace) is chosen to be scheduled first and independently of other paths, then another trace is chosen and scheduled, and so on. TS doesn't allow certain types of code motion across the main trace.

In [17] an approach is presented where code-motions are exploited. BBs are scheduled using a list scheduler and then code motions are allowed. One priority function is used in the BB scheduler and another for code motion. Code motion is allowed only inside windows containing a few BBs to keep runtime low, but then iterative improvement is needed not to restrict too much the kind of code motions allowed.

Among those methods, only PBS is exact, but it solves a partial problem where speculative execution is not allowed. TBS and CVLS address BB scheduling and code motions simultaneously, but use classical list scheduler heuristics. TS combines both subproblems in a per trace basis, but maintrace-first heuristics are applied. In [17] a different heuristic is applied to each subproblem. All those methods may exclude optimal solutions from the search space.
In [16], an exact symbolic technique for control dependent scheduling is presented. However, restrictions are imposed on the speculative execution model. Besides, the use of an exact method in early (more iterative) phases of a design is unlikely, especially because no pruning is presented to cope with the larger search space due to code motions.

A method could be envisaged where no restriction is imposed neither on the kind of code motion, nor on the order the operations are taken to be scheduled.

\section{The method}

\subsection{Outline of the method}

The solution constructor takes a permutation of the operations and generates a solution. Techniques borrowed from the constructive topological sorted scheduler [8] are used, because it has the important property that there always exists a permutation which results in an optimal solution. A schedule is constructed out of the permutation as follows. An operation to be scheduled is selected among ready operations (unscheduled operations whose predecessors are all scheduled) following the order in the permutation. Each selected operation is attempted to be scheduled at the as early as possible time where a free resource is available.

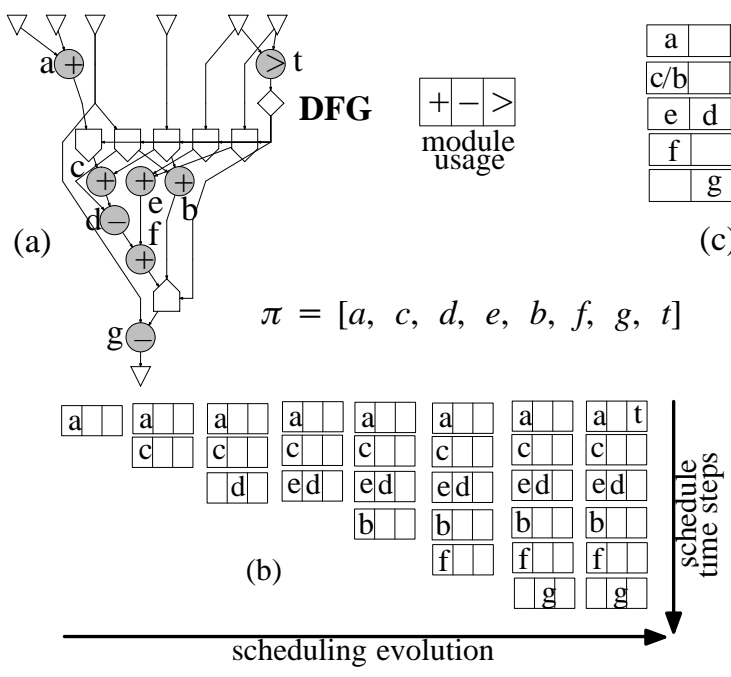

\section{Figure 2 - Using the topological sorted scheduler}

In figure 2, a linear-time sequence is constructed for a given permutation. See [5] for an explanation of the symbols used. The topological sorted scheduler is applied on purpose in a very straightforward way (figure $2 b$ ). No information about mutual exclusion is used. When such information is used, $b$ can be scheduled at the second step by sharing an adder with operation $c$ (figure $2 \mathrm{c}$ ). We are assuming that the outcome of $t$ is not available inside the first step to allow $a$ and $b$ to conditionally share a resource. The resulting schedule length is reduced to 5 steps for both EXIs, even though the EXI $(t, b, g)$ could be scheduled on its own in only two steps. Information about mutual exclusion is clearly not enough and the limitation is the linear-time model. To allow a more efficient solution, some mechanism has to split the lineartime sequence by exposing a flow of control. Our mechanism is based on initial links, as we will explain in the next subsections. 


\subsection{Initial links}

In our method we want to capture the freedom for code motions without restrictions and for this purpose we introduce the notion of a link. A link connects an operation $u$ in the DFG with a BB $v$ in the BBCG. Its interpretation is that $u$ may be executed under the predicate which defines the execution of operations in $v$. A same operation can be linked to several mutually exclusive BBs. Figure 3 illustrates the link concept. A merge node (M) represents data selection and a branch node (B) represents control selection.

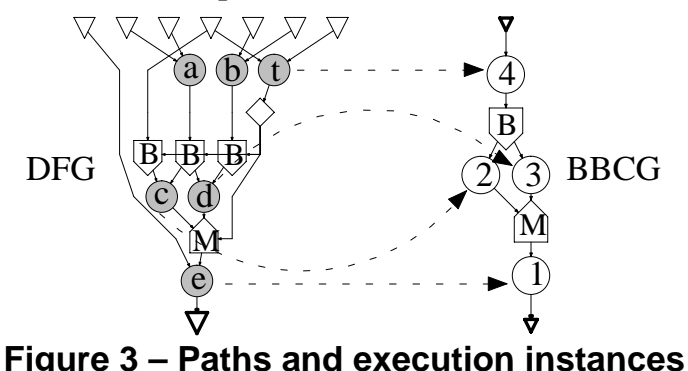

Figure 3 - Paths and execution instances

Initial links: We will encode the freedom for code motion by using a set of initial links. First, we look for each operation whose result has to be available before a control or data selection point in a given EXI. Such an operation is said to be a terminal. Each terminal is linked to the $\mathrm{BB}$ which precedes the corresponding selection point in the BBCG. In figure 3, initial links are shown for terminals $c$ and $d$ (due to data selection) and $t$ (due to control selection).Then, we link each ancestor of a terminal to the same BB to which the latter is linked. Operation $a$ is not a terminal and will have initial links (not shown in figure) to both $\mathrm{BB} 2$ and $\mathrm{BB} 3 ; b$ will have a single initial link pointing to BB3. Each link points to the latest $\mathrm{BB}$ in a given path where the respective operation can still be executed. This means that each operation is free to be executed inside any preceding BB on the same path as soon as data precedence and resource constraints allow (the only control dependency to be satisfied is the need to execute the operation at the latest inside the $\mathrm{BB}$ pointed by the initial link). The underlying idea is to traverse the BBCG in topological order trying to schedule operations in traversed BBs. If operation $u$ is given an initial link to BB $v$ and $v$ is reached in the traversal, then $u$ must be scheduled inside it. We say that the assignment of $u$ to BB $v$ is compulsory.

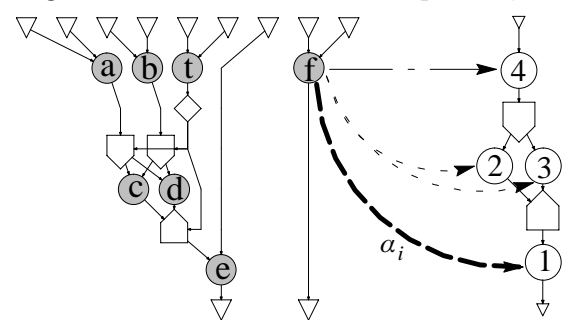

Figure 4 - Linking unconditional operations

Final assignments: Each link $\alpha$ will be called here an operation assignment (from now on called simply assignment) when it obeys precedence constraints and it doesn't imply the need for more than the available functional units. Assignments which might increase registers and/or interconnect usage are included in the search space. Each assignment from an operation $u$ into a $\mathrm{BB} v$ is given the following attributes: a) condition (execution condition of $u$ when its predicate is completely evaluated); b) begin (operation starting time of $u$ in BB $v$ ); c) end (completion time of $u$ in BB $v$ ). Assignments represent a relative-time encoding. The absolute time is given by the instant BB $v$ starts execution plus the value in attribute begin.

Freedom for code motion: Operations may be redundant for some paths in a behavioral description [10]. TBS uses tree optimization to remove redundancies by propagating each operation to the latest BB where it is to be used. CVLS eliminates them by using extended condition vectors [18]. Even though they remove redundancies, those methods don't succeed to encode the freedom for code motion. Tree optimization works well for (nested) conditional trees, but in the case of parallel flows of execution, the quality of a solution may depend on the description. In figure 4 , after tree optimization, the unconditional operation $f$ would remain associated to the $\mathrm{BB}$ wherein it was originally described.

Merging concurrent flows of execution: Our initial links do not only handle redundancies, but also encode freedom for code motion. In figure $4, f$ may be linked to BB4, to both $\mathrm{BB} 3$ and $\mathrm{BB} 2$ or to BB1. $\alpha_{i}$ is the initial link, because the only control dependency to be satisfied is that $f$ execute before the output is available. As operation $f$ can be executed in BB 1 or in any preceding $\mathrm{BB}$ as soon as resource and data dependency constraints are satisfied, unrestricted code motions can be exploited, even for concurrent flows of execution.

\subsection{The solution constructor}

Traversing in topological order: The solution constructor follows the flow of tokens in the DFG while the BBCG is traversed in topological order. An operation can be assigned to any traversed $\mathrm{BB}$, as soon as data precedence and resource constraints allow. If more than one operation satisfies these constraints, an operation will be chosen based on the order in the permutation. Such an assignment is not compulsory as long as the $\mathrm{BB}$ to which the operation was initially linked is not reached. As a result, an initial link $u \rightarrow v$ might become a final assignment, but it will be revoked if $u$ succeeds to be scheduled inside any ancestor of $v$, inducing a code motion.

Splitting the linear-time sequence: Only when an operation is compulsory in a BB, it is allowed to "allocate" extra time steps inside that BB. This will make room for scheduling non-compulsory operations in idle resources. We claim that the underlying pruning associated with this criterion to split the linear-time sequence doesn't discard any better solutions (see section 2 and proof in Appendix A).

Example: In figure 5 the same example as in figure 2 is scheduled to illustrate the method. It is shown in figure $5 \mathrm{~b}$ how each EXI could be scheduled independently. EXI1 $=\{\mathrm{t}$, $\mathrm{a}, \mathrm{c}, \mathrm{e}, \mathrm{d}, \mathrm{f}, \mathrm{g}\}$ is scheduled in five steps and EXI $2=\{\mathrm{t}, \mathrm{b}, \mathrm{g}\}$ is scheduled in 2 steps. It is not possible to overlap those sequences, because $a$ and $b$ cannot share the adder (the outcome of test $t$ is not available inside the first step). Even though each path can be AFAP scheduled for the given $\Pi$, there is a conflict between them so that if one sequence is chosen, the other will be imposed an extra step. Figure 5c shows the initial links. Figures $5 \mathrm{~d}$ to $5 \mathrm{k}$ show the evolution 


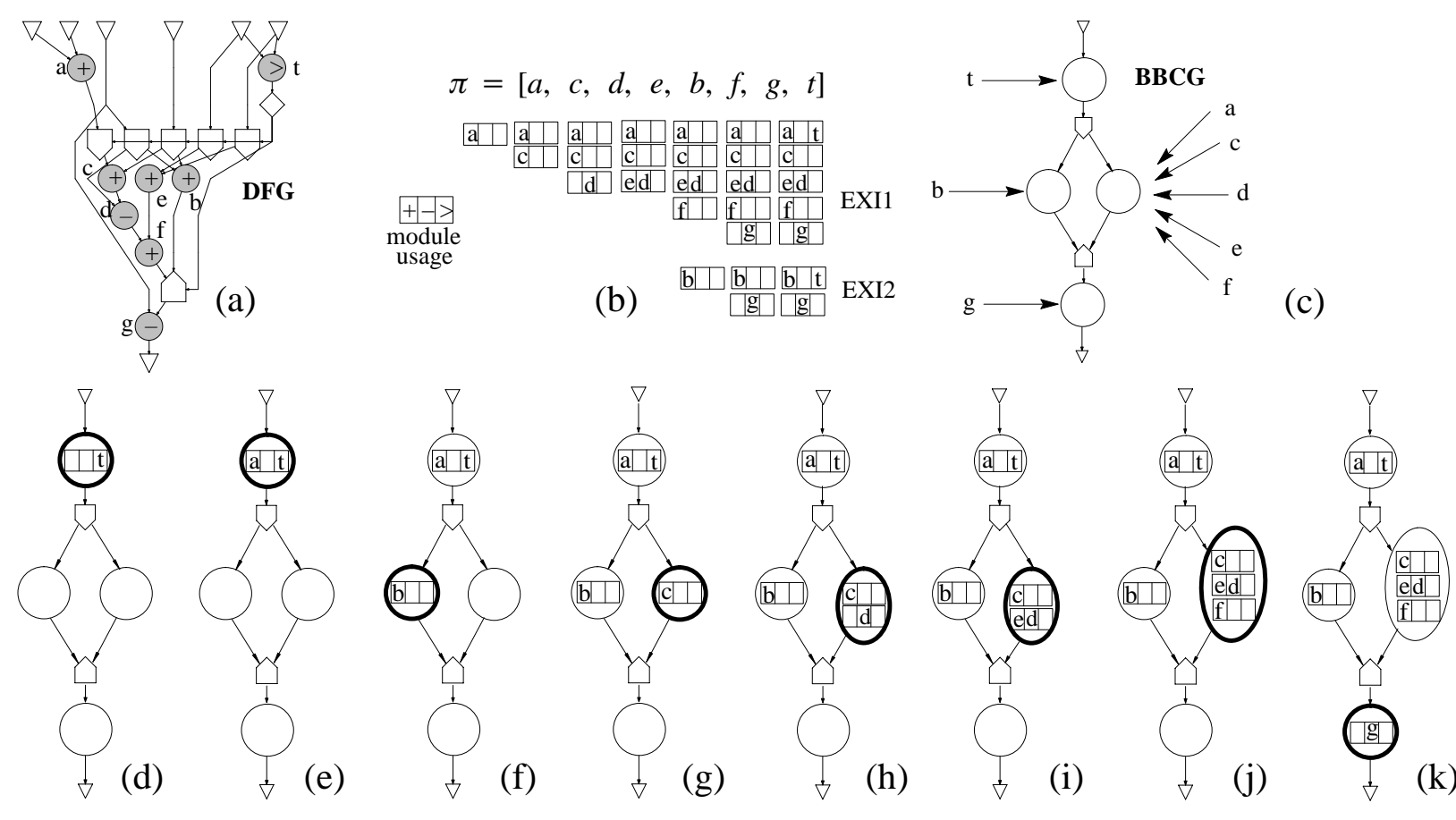

Figure 5-Splitting the linear-time sequence

for each operation in $\Pi$. Circles in bold mark the current BB being traversed. Note in figure $5 \mathrm{~d}$ that, even though other ready operations ( $a, e$ and $b$ ) precede $t$ in $\Pi, t$ is the scheduled one because it is the only compulsory operation in the current $\mathrm{BB}$. Then $a$ is scheduled (figure 5e) in the same step, as an idle adder exists. At that point no other ready operations can be scheduled in that $\mathrm{BB}$, as they would require the allocation of extra steps. Then, another BB is taken (figure 5f) and so on. Figure $5 \mathrm{k}$ shows the final result. It is the same as obtained by scheduling EXI1 independently (figure 5b), but EXI 2 was imposed an extra step. Note that if $a$ and $b$ were exchanged in $\Pi$, the solution in $5 \mathrm{~b}$ for EXI 2 would be obtained, while EXI1 would be imposed an extra step. When a conflict happens between paths, the method solves it in a certain way induced by $\Pi$, but there exists another permutation $\Pi^{\prime}$ which induces a solution in the opposite way (no limitation in search space). Note that the assignment of operations $a$ or $b$ to the first step represents speculative execution. If we don't allow speculative execution both EXIs will be imposed an extra step, resulting in schedule lengths of 3 and 6.

Notion of order dominant over notion of time step: As opposed to other approaches [18, 12], this method doesn't use time as a primary issue to decide on the position an operation will be assigned to. Instead, a notion of order and availability of resources in control flow is used. As assignments incorporate a relative-time encoding, time is only used to manage resource utilization inside $\mathrm{BBs}$. Our approach doesn't enumerate exhaustively combinations of time steps to schedule an operation, which exempts the use of greedy choices to decide on the position of an operation in different paths [12] and to control the number of states [18].

The solution constructor is summarized in algorithm $1 . \Pi$ is a permutation, $C$ is the set of BBs, $u$ is an operation, $v$ is a BB, $\alpha$ is an assignment and $\sigma(\alpha)$ is a real number. Function
$\operatorname{maxTop}(C)$ returns BBs in a arbitrary topological order. A candidate assignment $\alpha$ is created for each pair $(u, v)$ and the condition unscheduled $(\alpha) \wedge$ scheduledpreds $(\alpha)$ is evaluated. If this condition holds, the earliest step in $v$ with a free resource $(\sigma(\alpha))$ will be found. Function isSuitable $(\alpha, \sigma(\alpha))$ decides whether $\alpha$ should be committed or revoked. When all compulsory operations are scheduled and there is no room for scheduling others, a new BB is taken.

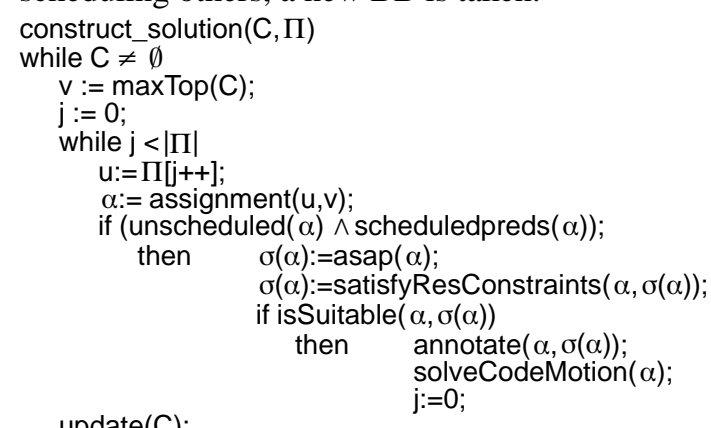

\section{Algorithm 1 - The solution constructor}

Runtime complexity: Let $n$ be the number of operations in $\Pi, b$ the number of BBs, $p$ the number of paths and $c$ the number of conditionals. The search for the first ready operation in $\Pi$ takes $O(\lg n)$. As this search may be repeated for each operation and for each BB, the worst case complexity of algorithm 1 is $O(b n \lg n)$. The runtime efficiency of our approach doesn't depend on $p$ (which can grow exponentially in $c$ ), as opposed to path-based methods.

\subsection{Exploiting unrestricted code motions}

In this section we summarize the relationship between initial links, assignments and code motions. A detailed analysis of code motions can be found in [17]. 


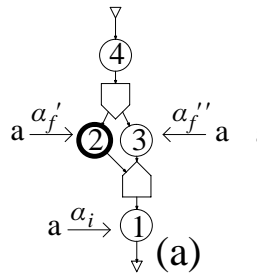

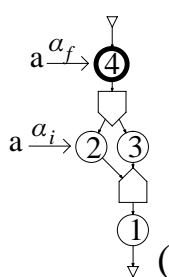
(b)

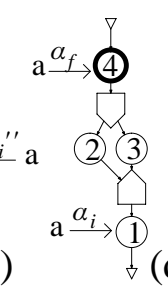

(d)

Figure 6 - Basic code motions

Basic code motions: Figure 6 illustrates code motions in the scope of a single conditional: duplication-up (a), boosting-up (b), unification (c) and useful (d). $\alpha_{f}$ represents a final assignment and $\alpha_{i}$ an initial link. A circle in bold represents the current BB being traversed. Once $\alpha_{f}$ succeeds, the covered $\alpha_{i}$ 's are revoked. Even though only upward motions are explicitly shown, downward motions are implicitly supported, as the initial links encode the maximum freedom for code motions downwards.
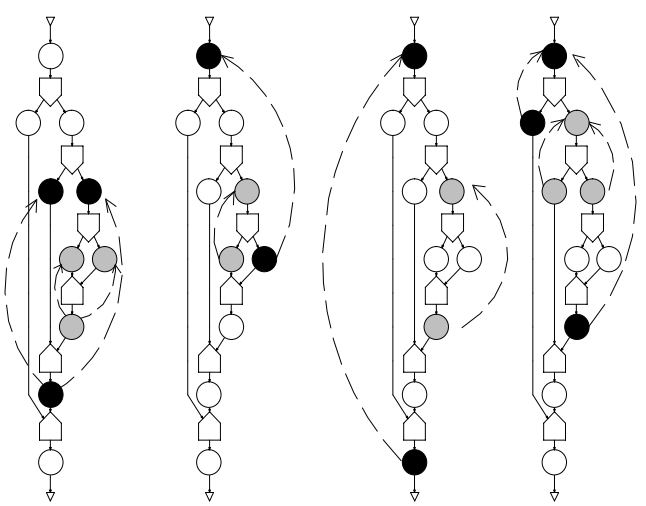

Figure 7 - Generalized code motions

Generalized code motions: Figure 7 shows generalized code motions supported in our approach. Arrows indicate possible upward motions from an origin BB to a destination BB. Gray circles illustrate more local code motions, which are handled by most methods. Either they correspond to the basic code motions of figure 6 or to a few combinations of them. In [17] these combinations are attempted via iterative improvement inside windows containing a few BBs. Black circles illustrate more global code motions also supported in our method. Note that such "compound" motions are determined at once by the permutation and are not the result of successive application of basic code motions. We do not search for the best code motions inside a solution, we do search for the best solution whose underlying code motions induce the best overall cost.

Impact of code motions on the search space: As any assignment determined by a permutation may induce a code motion, unrestricted types of code motions are possible. As a result, the search space is not limited by any restriction on the nature, amount or scope of code motions. This is convenient in the late phases of a design, when optimization goals include usage of registers and interconnect and number of states (which might all be affected by code motions).

Code-motion pruning: However, in early phases of a design, we need a fast but accurate estimate only in terms of schedule length $[4,9]$. In this scenario, we can allow some reduction in search space by pruning code motions which

guaranteedly don't induce better solutions (see Appendix A).

Impact on different application domains: Control- dominated applications normally require that each path be optimized as much as possible. Here the role of code motion is obvious. However, in DSP applications, it is unnecessary to optimize beyond the given global time constraint [14]. Even though highly optimized code might not be imperative, the role of code motions should not be overlooked even in DSP applications, because code motions can reduce the schedule length of the longest control path. The tighter the constraints are, the more important the code motions become. In our approach, the advantage of taking code motions into account is not bestowed at the expense of a "much larger search space", due to our code-motion pruning. Code motion is especially important when simple controllers are used for code retargetability [11].

\section{Experimental results}

The method has been implemented in the NEAT system [7]. A genetic algorithm is used as solution explorer in the current implementation. For the representation of predicates we are using the BDD package developed by Geert Janssen.

Table 1 - Results for Wakabayashi's example

\begin{tabular}{|c|c|c|c|c|c|c|}
\hline method & case & \#alu & \#add & \#sub & chain & lengths \\
\hline \multirow{2}{*}{ ours } & a & 0 & 1 & 1 & 1 & $4,4,7$ \\
& b & 0 & 1 & 1 & 2 & $3,4,7$ \\
& c & 2 & 0 & 0 & 2 & $3,4,6$ \\
\hline \multirow{2}{*}{ TBS } & a & 0 & 1 & 1 & 1 & $4,4,7$ \\
& b & 0 & 1 & 1 & 2 & $3,4,7$ \\
& c & 2 & 0 & 0 & 2 & $3,4,6$ \\
\hline \multirow{2}{*}{ HRA } & a & 0 & 1 & 1 & 1 & $4,4,7$ \\
& b & 0 & 1 & 1 & 2 & $3,4,7$ \\
& c & 2 & 0 & 0 & 2 & $3,5,6$ \\
\hline CVLS & a & 0 & 1 & 1 & 1 & $4,5,7$ \\
\hline \multirow{2}{*}{ PBS } & b & 0 & 1 & 1 & 2 & $3,6,7$ \\
& c & 2 & 0 & 0 & 2 & $3,5,6$ \\
\hline
\end{tabular}

Table 2 - Results for maha and parker

\begin{tabular}{|c|c|c|c|c|}
\hline \multirow{2}{*}{ method } & \multicolumn{2}{|c|}{ maha } & \multicolumn{2}{c|}{ parker } \\
\cline { 2 - 5 } & max/min & avg & max/min & avg \\
\hline ours & $4 / 1$ & 2.25 & $4 / 1$ & 2.00 \\
\cline { 1 - 1 } CVLS & $4 / 1$ & 2.38 & $4 / 1$ & 2.38 \\
\cline { 1 - 1 }$[16]$ & $4 /-$ & 2.25 & $4 /-$ & 2.13 \\
\hline \multicolumn{3}{|c}{ \#adders = 2; \# subtractors = 3 } \\
\hline
\end{tabular}

In table 1, our method is compared with others for the example in [18]. Results were collected from [10], [18] and [12]. Our solution for case $a$ is as good as TBS and HRA[12] and better than CVLS. In case $b$ our method, TBS and HRA reach the same results which are better than PBS. For case $c$, both our method and TBS are better than HRA and PBS. In table 2 we compare our results for benchmarks maha and parker used in [19] and [16]. Our method reaches the same average values for those methods during exploration $(2.25$, 2.38 and 2.13), but a better average value (2.00) is found.

Although we certainly need to perform more experiments, these first results are encouraging. They seem to confirm that our method is able to find the code motions which induce the better solutions.

Figures 8 and 9 show the impact of our code-motion pruning. For the experiment, 50 solutions were constructed 
induced by randomly generated permutations. Figure 8 shows results with (black) and without (gray) pruning. The height of a bar represents the number of solutions counted under different assumptions. In case (3) (waka(3) and maha(3)), solutions are distinguished by comparing the length of each path. In cases (1) and (2), solutions are distinguished only by their overall cost. Case (1) uses $\max T_{i}$ as a cost function and case (2) uses $\sum T_{i}$. In waka(3) for example, 32 different solutions are identified without pruning, but only 5 with pruning. This reduction will lead to an effective reduction in search space, which depends on the chosen cost function (compare waka(1) and (2)).

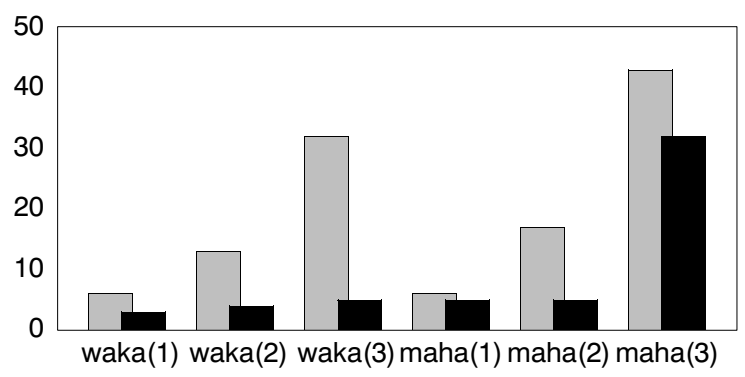

Figure 8 - Reduction of the number of solutions

Not only the number of solutions is reduced, but also the range of cost values. The cost range ratio "no pruning/pruning" is 2.5 for waka(1) and maha(1), 3.5 for waka(2), and 1.55 for maha(2). These ratios are shown in figure 9, normalized with respect to the "no pruning" cost range. This compaction on cost range suggests a higher probability of reaching (near) optimal solutions during local search.

\section{Conclusions and Future Work}

We have cast the resource-constrained OP for descriptions with conditionals into a local search problem. The permutation-driven constructor deals with code motions constructively while keeping optimal solutions in the search space. The approach can be extended to include other issues.

Most methods for conditionals either break loops or allow limited optimization between iterations. Our method could accommodate one of such approaches, but we prefer to investigate loops as a further topic to prevent limitation on the search space. We also intend to consider time constraints.

\section{Appendix A. Proof of the pruning technique}

Theorem: Let $S_{m}$ be a solution of the described OP constructed with algorithm 1 for a given $\Pi$ and consider an operation $o$ assigned to $\mathrm{BB} j$ in such a solution. Let $\delta$ be $[\operatorname{delay}(o)]$. If a solution $\mathrm{S}_{\mathrm{n}}$ is obtained by moving $o$ from BB $j$ into BB $i$ and it allocates exactly $\delta$ cycle steps inside $\mathrm{BB} i$, then $\operatorname{cost}(\mathrm{Sn}) \geq \cos t(\mathrm{Sm})$, where $\cos t$ is a monotonically increasing function in terms of schedule lengths. Proof: Let $l(k), L(k) \in \mathbb{N}$ be the schedule lengths of a BB $k$ before and after the motion, respectively. Let $p, q, r, s$ be BBs forming path $_{\mathrm{i}}: p \rightarrow q \rightarrow r$ and path $: p \rightarrow s \rightarrow r$. Let $l_{n}$ and $L_{n}$ be, respectively, the schedule lengths of path ${ }_{n}$ before and after motion.

a) $o$ was assigned to $q$ and allocates $\delta$ steps inside $p \Rightarrow L(\mathrm{p})=l(\mathrm{p})+\delta$ a.1) No operation assigned to $r$ can be moved in the allocated steps $\Rightarrow L(r)=l(r), L(\mathrm{~s}) \geq l(\mathrm{~s})-\delta, L(\mathrm{q}) \geq l(\mathrm{q})-\delta \Rightarrow L_{i} \geq l_{i}$ and $L_{j} \geq l_{j}$ a.2) There is an operation $u$ assigned to $r$ which can be moved in the allocated steps. As $u$ depends or has resource conflicts with operations assigned to $\mathrm{q}$ and $\mathrm{s}$ (topological sorted construction)
$\Rightarrow L(\mathrm{q}) \geq l(\mathrm{q})-\delta+\lceil\operatorname{delay}(u)\rceil, L(\mathrm{~s}) \geq l(\mathrm{~s})-\delta+\lceil\operatorname{delay}(u)\rceil$,

$L(\mathrm{r}) \geq l(\mathrm{r})-\lceil\operatorname{delay}(u)\rceil \Rightarrow L_{i} \geq l_{i}$ and $L_{j} \geq l_{j}$

b) $o$ was assigned to $r$ and allocates $\delta$ steps inside both $q$ and $s$ $\Rightarrow L(\mathrm{q})=l(\mathrm{q})+\delta, L(\mathrm{~s})=l(\mathrm{~s})+\delta, L(\mathrm{r}) \geq l(\mathrm{r})-\delta \Rightarrow L_{i} \geq l_{i}$ and $L_{j} \geq l_{j}$ c) $o$ was assigned to $r$ and allocates $\delta$ steps inside $p \Rightarrow L(\mathrm{p})=l(\mathrm{p})+\delta$ $\Rightarrow o$ doesn't depend but has resource conflicts with operations assigned to $\mathrm{q}$ and $\mathrm{s}$ (topological sorted construction)

$\Rightarrow \mathrm{L}(\mathrm{q})=\mathrm{l}(\mathrm{q}), L(\mathrm{~s})=l(\mathrm{~s}), L(\mathrm{r}) \geq l(\mathrm{r})-\delta \Rightarrow L_{i} \geq l_{i}$ and $L_{j} \geq l_{j}$

For a given $\Pi$, solution $S_{\mathrm{n}}$ have path lengths greater than or equal to those in $\mathrm{S}_{\mathrm{m}}$. As compound code motions can be built out of these basic code motions, and cost is monotonically increasing, we can conclude without loss of generality that $\operatorname{cost}(\mathrm{Sn}) \geq \operatorname{cost}(\mathrm{Sm})$.

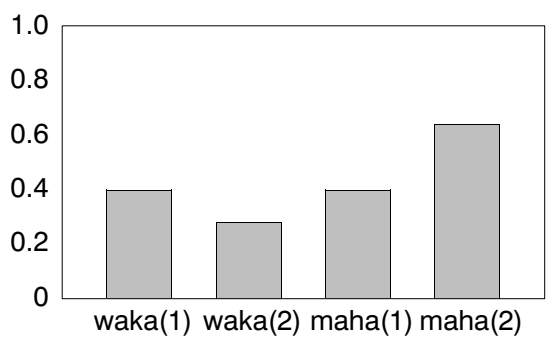

Figure 9 - Compaction on cost range

\section{References}

[1] S. Amellal and B. Kaminska, "Functional Synthesis of Digital Systems with TASS," IEEE Trans. CAD, 13(5): 537-552, May 1994. [2] R. Bergamaschi et al., "Area and Performance Optimizations in Path Based Scheduling," in Proc. Europ. Conf.on Des. Automation, pp. 304-310, 1991.

[3] R. Camposano, "Path-based scheduling for synthesis," IEEE Trans. on CAD, 10(1): 85-93, Jan. 1991.

[4] R. Camposano and J. Wilberg, "Embedded System Design, " Design Autom. for Embedded Syst. Journal, n. 1, pp. 5-50, 1996.

[5] J.Eijndhoven and L.Stok, "A Data Flow Exchange Standard," in Proc. Europ. Conf. on Des. Automation, pp. 193-199, 1992.

[6] J. A. Fisher,"Trace Scheduling: A technique for global microcode compaction," IEEE Trans. Comput., vol. 30, July 1981.

[7] M.Heijligers et al., "NEAT: an Object Oriented High Level Synthesis Interface", in Proc. IEEE ISCAS'94., pp.1.233-1.236,1994. [8] M.Heijligers and J.Jess, "High-Level Synthesis Scheduling and Allocation using Genetic Algorithms based on Constructive Topological Scheduling Techniques," Int. Conf. Evol. Comp.,1994 [9] J. Henkel and R. Ernst. "A Path-based Technique for Estimating Hardware Runtime in HW/SW-cosynthesis", in Proc. ACM/IEEE ISSS'95, pp.116-121.

[10] S.Huang et al.," A tree-based scheduling algorithm for control dominated circuits," in Proc. ACM/IEEE DAC'93, pp-578-582

[11] A.Kifli et al.,"A Unified Scheduling Model for High-Level Synthesis and Code Generation," in Proc. ED\&TC'95, pp.234-238.

[12] T. Kim et al.," A Scheduling Algorithm for Conditional Resource Sharing - A Hierarchical Reduction Approach", IEEETrans. on CAD, 13(4):425-438, Apr 1994.

[13] M. S. Lam and R. P. Wilson, "Limits of Control Flow on Parallelism," ACM/IEEE Int. Symp. Comput.Archit., 1992,pp.46-57. [14] R.Leupers andP.Marwedel,"'Time constrained CodeCompaction for DSPs", in Proc. ACM/IEEE ISSS'95, pp.54-59.

[15] C. Papadimitriou and K. Steiglitz." Combinatorial optimization: algorithms and complexity". Prentice Hall, 1982.

[16] I.Radivojevic and F.Brewer, "A New Symbolic Technique for Control Dependent Scheduling,"IEEETrans.CAD,15(1):45-57, 1996. [17] M. Rim, et al., "Global Scheduling with Code-Motions for High-Level Synthesis Applications," IEEE Trans. on VLSI Systems, vol. 3, no. 3, Sept. 1995, pp. 379-392.

[18] K. Wakabayashi and T. Yoshimura, "A resource sharing and control synthesis method for conditional branches," in Proc. ACM/IEEE ICCAD'89, pp.62-65.

[19] K. Wakabayashi and H. Tanaka,"Global scheduling independent of control dependencies based on condition vectors," in Proc. ACM/IEEE DAC'92, pp.112-115. 